\title{
Decline of lymphatic vessel density and function in murine skin during aging
}

\author{
Sinem Karaman ${ }^{1} \cdot$ Dorina Buschle $^{1} \cdot$ Paola Luciani $^{1} \cdot$ Jean-Christophe Leroux $^{1} \cdot$ \\ Michael Detmar ${ }^{1} \cdot$ Steven T. Proul $^{1}$
}

Received: 20 March 2015 / Accepted: 25 July 2015/Published online: 11 August 2015

(C) Springer Science+Business Media Dordrecht 2015

\begin{abstract}
Lymphatic vessels play important roles in the pathogenesis of many conditions that have an increased prevalence in the elderly population. However, the effects of the aging process on the lymphatic system are still relatively unknown. We have applied non-invasive imaging and whole-mount staining techniques to assess the lymphatic vessel function and morphology in three different age groups of mice: 2 months (young), 7 months (middleaged), and 18 months (aged). We first developed and validated a new method to quantify lymphatic clearance from mouse ear skin, using a lymphatic-specific near-infrared tracer. Using this method, we found that there is a prominent decrease in lymphatic vessel function during aging since the lymphatic clearance was significantly delayed in aged mice. This loss of function correlated with a decreased lymphatic vessel density and a reduced lymphatic network complexity in the skin of aged mice as compared to younger controls. The blood vascular leakage in the skin was slightly increased in the aged mice, indicating that the decreased lymphatic function was not caused by a reduced capillary filtration in aged skin. The decreased function of lymphatic vessels with aging might have implications for the pathogenesis of a number of aging-related diseases.
\end{abstract}

Electronic supplementary material The online version of this article (doi:10.1007/s10456-015-9479-0) contains supplementary material, which is available to authorized users.

Steven T. Proulx

steven.proulx@pharma.ethz.ch

1 Institute of Pharmaceutical Sciences, Swiss Federal Institute of Technology, ETH Zurich, Vladimir-Prelog-Weg 1-5/10, 8093 Zurich, Switzerland
Keywords Aging - Lymphatic vessels · Vascular leakage $\cdot$ In vivo imaging

\section{Introduction}

The lymphatic vascular system is responsible for the maintenance of tissue fluid homeostasis, the migration of immune cells to secondary lymphatic organs and the uptake of dietary fat from the intestine [1]. The lymphatic vasculature is also critical in pathological situations such as the spread of cancer cells from solid tumors, the development of lymphedema, and the resolution of chronic inflammation [2]. Recently, it has been discovered that lymphatic vessels in the skin also play key roles in reverse cholesterol transport [3,4] and salt uptake for blood pressure regulation [5], findings that may have clinical implications for heart disease.

Despite the increased prevalence of lymphatic-related diseases with age, our knowledge about how the lymphatic system is affected by the aging process is still limited. Previous studies have suggested that lymphatic function might decline with age [6]. Two studies used lymphoscintigraphy in elderly patients with cancer and venous disease and found a decreased nodal uptake of lymphoscintigraphic tracers in aged individuals [7, 8]. More recent reports indicate that collecting lymphatic vessels in aged rats have a reduced pumping efficiency [9-11]. A recent clinical study supported this finding, using a pressure cuff technique and near-infrared (NIR) imaging with indocyanine green dye to find a reduced pumping strength of collecting lymphatic vessels in the leg of aged individuals [12]. However, a study combining functional and morphological data to evaluate the effect of aging on dermal lymphatic vessels has not yet been performed. 
In order to assess how lymphatic function is altered during aging, new methods are needed that can accurately quantify the function of the lymphatic vascular system in a non-invasive manner. A clinical method that can track lymphatic removal of an interstitial depot of radiolabeled tracer was developed for assessment of the lymphatic function in patients with lymphedema [13]. This approach was adapted for use in rodent models using a fluorescent dye bound to human albumin [14]. We have recently developed polyethylene glycol (PEG)-conjugated NIR fluorescent tracers of sufficient molecular weight such that they are specifically taken up by the lymphatic vessels after intradermal injection with no uptake visualized in the venous system [15]. The fluorescent dye remains stably bound to the PEG molecule in vivo and the tracers do not demonstrate accumulation in the draining lymph nodes through phagocytosis [2]. Therefore, these tracers have great potential to quantify lymphatic clearance by monitoring the disappearance of fluorescent signal at the site of injection.

In the current study, we established and validated a new assay for measuring lymphatic clearance from mouse skin. Using this assay, we demonstrated that there is a steady decline in the lymphatic clearance rate with age. This decline is associated with a reduction in lymphatic vascular density and complexity in the skin.

\section{Methods}

\section{Mice}

C57BL/6 J-Tyr ${ }^{c-J}$ albino mice (Jackson Laboratories) and C57BL/6C mice (Charles River) were maintained under pathogen-free conditions until imaging. K14-VEGF-C and K14-sVEGFR-3-Fc mice on an FVB background were a kind gift of Dr. Kari Alitalo, University of Helsinki [16, 17]. After imaging, the mice were euthanized with an overdose of anesthesia (1000 mg/kg ketamine; $3.5 \mathrm{mg} / \mathrm{kg}$ medetomidine) followed by cervical dislocation. All methods were carried out in strict accordance with the approved guidelines and all experiments were performed in accordance with animal protocols approved by Kantonales Veterinaramt Zurich (protocol: 237/2013).

\section{Lymphatic-specific imaging tracers}

The PEG-based lymphatic tracer P20D800 was prepared by reacting methoxypoly(ethylene glycol) amine P20 (20 kDa) equimolarly with IRDye ${ }^{\circledR} 800 \mathrm{CW}$ NHS Ester (LI-COR Biosciences, Lincoln, NE) in anhydrous dimethyl sulphoxide. The crude reaction was then diluted with $4 \mathrm{~mL}$ of ultrapure water and freeze-dried overnight to ensure complete removal of the organic solvent. The dry lyophilizate was then reconstituted with $250 \mu \mathrm{L}$ of HEPES buffered saline (20 mmol/L HEPES, $145 \mathrm{mmol} / \mathrm{L} \mathrm{NaCl}, \mathrm{pH} 7.4$ ) and purified, as previously described [18]. The stock solutions of the polymeric dyes were kept at $-20{ }^{\circ} \mathrm{C}$ until further use.

\section{Stability of P20D800 in lymph}

In order to check the stability of the tracer in lymph, a P20D800 stock solution in HEPES buffered saline was incubated at $37{ }^{\circ} \mathrm{C}$ for $24 \mathrm{~h}(7.5 \mu \mathrm{mol} / \mathrm{L}$ D800 concentration in lymph) under constant shaking. At allotted time points aliquots were collected, deproteinized, and analyzed via HPLC.

Deproteinization of P20D800-containing lymph was achieved following an optimized method that allowed $100 \%$ dye recovery from lymph [19]. Briefly, a volume of $20 \mu \mathrm{L}$ of dye-containing lymph was added to $30 \mu \mathrm{L}$ of acetonitrile/methanol $(47 / 3 \mathrm{v} / \mathrm{v})$. The tube was vortexed and centrifuged for $10 \mathrm{~min}$ at $1500 \times \mathrm{g}$. The supernatant was withdrawn, and eluted in an analytical HPLC following the gradient protocol (\% A/B): $90 / 10$ to $50 / 50$ for $10 \mathrm{~min}$, isocratic $50 / 50$ for $5 \mathrm{~min}, 50 / 50$ to $10 / 90$ for $10 \mathrm{~min}$, isocratic $10 / 90$ for $5 \mathrm{~min}, 10 / 90$ to $90 / 10$ for $5 \mathrm{~min}$, where solvent A consisted of $10 \mathrm{mmol} / \mathrm{L}$ tetrabutylammonium bromide (Sigma) aqueous solution and solvent $\mathrm{B}$ of $10 \mathrm{mmol} / \mathrm{L}$ tetrabutylammonium bromide in acetonitrile/water $(90 / 10 \mathrm{v} / \mathrm{v})$. The flow rate of the mobile phase was $1.0 \mathrm{~mL} / \mathrm{min}$. The detector wavelength was set at $780 \mathrm{~nm}$. The chromatographic column used was a YMC Pack Pro C18 $150 \mathrm{~mm} \times 4.6 \mathrm{~mm}$ column with $3 \mu \mathrm{m}$ particles (YMC Co., Kyoto, Japan). Pure D800 carboxylate dye diluted in acetonitrile/methanol (47/3 v/v) was also eluted (7.5 $\mu \mathrm{mol} / \mathrm{L}$ D800 concentration in lymph) as control. The retention times for D800 and P20D800 were 19.3 and $13.9 \mathrm{~min}$, respectively.

\section{Lymphatic clearance assay}

Lymphatic vessel drainage function was assessed by measuring the clearance over time after intradermal skin injection of P20D800. Mice were anesthetized with isoflurane $(2 \%)$, and $3 \mu \mathrm{L}$ of $3 \mu \mathrm{mol} / \mathrm{L} \mathrm{P} 20 \mathrm{D} 800$ was injected into the ears with a $29-\mathrm{G}$ insulin syringe (Teramu, Somerset, NJ). The mice were then positioned in an IVIS spectrum (Caliper Life Sciences, Hopkington, MA) and an image was acquired with the following imaging settings: $\lambda_{\text {ex }}: 745 \mathrm{~nm}, \lambda_{\mathrm{em}}: 800 \mathrm{~nm}$, binning of 2 . An exposure time of $2 \mathrm{~s}$ was used for imaging in albino mouse strains, with an increased exposure time of $4 \mathrm{~s}$ for black mouse strains due to higher absorption from pigmentation and black fur. Subsequent images were acquired of the ears at 1, 2, 3, 4, 6, and $24 \mathrm{~h}$ after injection. Mice were allowed to wake up and 
move freely between imaging time points. Fluorescence signal intensities were adjusted to baseline ear signals before injection of tracers to calculate tissue enhancement values. Since there may be slight variations in the amount of tracer injected and in the initial distribution of the tracer after injection, we normalized the tissue enhancement values using a region of interest (ROI) encompassing the entire ear at all time points to the value directly after injection. The normalized fluorescence intensity values over time were fit to a one-phase exponential decay model in GraphPad Prism software with lymphatic clearance expressed as decay constant $\mathrm{k}$ (expressed in $\mathrm{h}^{-1}$ ) or as halflife (expressed in h) using the following equations:

Normalized Fluorescence Intensity $=\mathrm{e}^{-\mathrm{kt}}$,

Half Life $=\ln (2) / \mathrm{k}$.

For mice in which both ears were injected, values from each ear were averaged together to generate one decay constant $\mathrm{k}$ and half-life value per mouse.

\section{Evans blue lymphatic clearance assay}

Quantification of residual Evans blue dye in tissue after intradermal injection was performed as previously described [20]. Briefly, $3 \mu \mathrm{L}$ of $1 \%$ Evans blue dye (Sigma) was injected into the ear skin of isoflurane anesthetized mice. After $24 \mathrm{~h}$, the mice were sacrificed and the Evans blue dye was extracted from the tissue by incubation of the ears in formamide (Fluka). Absorbance was measured using an Infinite M200 microplate reader (Tecan, Männedorf, Germany) by measuring at $620 \mathrm{~nm}$. The concentration of dye in the tissue was calculated using a standard curve of Evans blue in formamide. Absorbance for P20D800 could not be detected using the microplate reader at $620 \mathrm{~nm}$.

\section{Ear lymphangiography}

High-resolution functional imaging to assess the spread of tracer within the dermal lymphatic network of the ear was performed after $3 \mu \mathrm{L}$ of $20 \mu \mathrm{M}$ P20D800 was injected using a 29-G insulin syringe (Teramu). Images at $200 \mathrm{~ms}$ exposure were acquired immediately after injection using a Zeiss StereoLumar microscope with AxioVision (Zeiss) software and a cooled EMCCD camera (Evolve eXcelon, Photometrics, Tuscon, AZ), light emitting diode (LED) system with illumination at $770 \mathrm{~nm}$ (CoolLED, Andover, UK) and specific filters for ICG (Semrock, Rochester, NY).

\section{Vascular leakage assay}

Vascular leakage was imaged and quantified as previously described [18]. In summary, vascular leakage was measured by dynamic imaging using an IVIS Spectrum in anesthetized C57BL/6 J-Tyr $r^{c-J}$ albino female mice $(\mathrm{n}=6$ young and $\mathrm{n}=5$ aged). A sequence of $3 \mathrm{~s}$ exposure images (excitation $745 \mathrm{~nm}$, emission $800 \mathrm{~nm}$, binning of 4) was initiated and paused after the first image was acquired. Then, $100 \mu \mathrm{L}$ per $20 \mathrm{~g}$ mouse weight of $10 \mu \mathrm{mol} / \mathrm{L} \mathrm{P} 20-\mathrm{D} 800$ tracer was injected intravenously into the tail vein and the imaging sequence was immediately resumed. Images were acquired every $15 \mathrm{~s}$ for a total imaging time of $15 \mathrm{~min}$. ROIs were drawn on each ear and the saphenous vein and the signal intensity values at each time point were determined using Living Image 4.0 software (Caliper). Leakage rates were determined by normalizing the signal intensity values of the ear ROIs to the value of the saphenous vein ROI at each time point to adjust for injected dose and the dynamics of intravascular signal. Blood volume fractions, tissue leakage rates, and vascular leakage rates were calculated as previously described [18].

\section{Whole mount immunostainings}

Whole mount immunostainings were performed as described [21]. Briefly, right ears were split in half and the inner part of the ear (without cartilage) was used for analyses. Lymphatic vessels were visualized using a rabbit-antimouse LYVE-1 (AngioBio, Cat no: 11-034, 1:600 dilution) as primary antibody. Alexa647 conjugated secondary antibody was purchased from Molecular Probes (Cat no: A-31573, 1:200 dilution). Thereafter, the samples were flat-mounted on glass slides using Mowiol (Calbiochem). Wide-field images of the whole ears at $6 \times$ and of a $34 \times$ region of interest close to the rim of the ear were obtained with a Zeiss StereoLumar microscope at $200 \mathrm{~ms}$ exposure with the Cy5 filter set. The acquired images were processed using Adobe Photoshop CS5 (Adobe Systems) and the LYVE-1 + area was analyzed with a self-prepared pipeline in CellProfiler software (version 2.1.0, BROAD Institute). Vessel length was measured in ImageJ (NIH) as described [22] and the number of branch points was counted manually.

\section{Statistical methods}

All data are shown as mean \pm SD. Means of two groups were compared using two-tailed Student's $t$ test; Welch's correction was used in case of unequal variances. Means of three groups were compared with one-way ANOVA with Tukey's multiple comparison post hoc test. All analyses were performed using GraphPad Prism V5.0 (GraphPad Software, San Diego, CA) or SPSS Statistics version 22 (IBM) and $P<0.05$ was accepted as statistically significant. 


\section{Results}

\section{Establishment and validation of a lymphatic clearance assay in mouse skin using NIR imaging tracers}

We first aimed to establish an in vivo lymphatic clearance assay using the NIR tracer P20D800 [18]. This tracer is comprised of the NIR dye IRDye800 bound to $20 \mathrm{kDa}$ PEG for an effective hydrodynamic diameter of about $8.4 \mathrm{~nm}$. We have previously shown specific uptake of the tracer by lymphatic vessels after interstitial injection [15]. P20D800 is stable in lymph as evidenced by the absence of cleavage of the fluorophore IRDye800 from the PEG polymer upon $24 \mathrm{~h}$ incubation at $37{ }^{\circ} \mathrm{C}$ (data not shown) and it is excreted intact by the kidneys after intravenous administration [18].

After interstitial injection, the removal rate of macromolecules by the lymphatic system has been found to follow a one-phase exponential decay model [13, 14]. With normalization of the fluorescence intensity in the tissue to the level directly after injection and the assumption that the signal will eventually decay to baseline fluorescence values, we hypothesized that the signal over a series of measurements should follow the model shown in Fig. 1a. For this model to be valid, however, the lymphatic tracer concentration should exhibit a linear relationship with fluorescence intensity. We therefore tested a dilution series of concentrations by using fluorescence measurements in an IVIS Spectrum imaging system and demonstrated a direct linear relationship (linear regression $\mathrm{r}^{2}=0.9958, P<0.0001$ ) of fluorescence intensity with concentrations up to $3 \mu \mathrm{M}$ (Fig. 1b). We therefore chose to inject a concentration of $3 \mu \mathrm{M}$ P20D800 for monitoring lymphatic clearance out of skin over time.

We next compared P20D800 clearance from the skin with the established Evans Blue assay, in which lymphatic function is estimated from the amount of residual injected dye in skin after a period of time by extracting the dye from the tissue of sacrificed animals [20]. We performed both assays in normal mice and in K14-VEGF-C transgenic mice that overexpress the lymphangiogenic growth factor VEGF-C in the skin [16] and that have previously been shown to have increased lymphatic function with the Evans Blue assay [20]. We injected $3 \mu \mathrm{L}$ of $1 \%$ Evans Blue dye into the skin of the left ear and $3 \mu \mathrm{L}$ of $3 \mu \mathrm{M}$ P20D800 into the right ear of 7-month-old male K14-VEGF-C transgenic mice and FVB wildtype littermates (Fig. 1c). Imaging in an IVIS spectrum revealed that immediately after injection of Evans Blue and P20D800, both tracers spread throughout the ear tissue of $\mathrm{K} 14-\mathrm{VEGF}-\mathrm{C}$ mice whereas the tracer was more localized in wildtype mice (Fig. 1d). We confirmed with high-resolution stereomicroscopy that the network of lymphatic vessels is vastly expanded in whole mounts of the skin of these mice as compared to wildtype mice and that after injection the tracer quickly spreads within the dermal lymphatic vessels in K14VEGF-C mice, as previously described [18, 23, 24] (Supplementary Fig. 1a, b online). By sequential IVIS imaging of a region of interest encompassing the entire ear, the loss of P20D800 fluorescence from the right ear was apparent using the NIR tracers, with enhanced disappearance in the K14VEGF-C mice (Fig. 1d, e). The fluorescence decay fit very closely to the one-phase exponential decay model, with a mean $r^{2}$ value of $0.987 \pm 0.012$ for the six animals studied. Quantification using the one-phase exponential decay model confirmed the significantly increased clearance in K14VEGF-C mice, as expressed by a reduced tissue half-life (wildtype $=3.2 \pm 0.2 \mathrm{~h} ; \quad \mathrm{K} 14-\mathrm{VEGF}-\mathrm{C}=2.2 \pm 0.1 \mathrm{~h}$; $P=0.003$; Fig. 1f) and an increased clearance rate (wildtype $=0.22 \pm 0.02 \mathrm{~h}^{-1} ; \mathrm{K} 14-$ VEGF-C $=0.32 \pm 0.02 \mathrm{~h}^{-1}$; $P=0.002$; Fig. 1g). Quantification of the Evans Blue dye extracted from the ears only revealed a non-significant trend towards increased clearance in the K14-VEGF-C mice (wildtype $=8.6 \pm 4.3 \mu \mathrm{g} / \mathrm{mL} ; \mathrm{K} 14-\mathrm{VEGF}-\mathrm{C}=4.4 \pm 1.1$ $\mu \mathrm{g} / \mathrm{mL} ; P=0.177$; Fig. 1h).

We next applied the NIR clearance assay to a model of diminished lymphatic function, namely the K14-sVEGFR-3Fc transgenic mice, which lack dermal lymphatic vessels [17, 25]. Injections of P20D800 were performed into both ears of 7-month-old male K14-sVEGFR-3-Fc mice $(n=6)$ and wildtype littermates $(n=5)$. Tracking and quantification of the fluorescent signal, using the IVIS Spectrum, revealed that more tracer remained in the ears of K14-sVEGFR-3-Fc mice compared to wildtype controls (Supplementary Fig. 2a, b online). The decreased lymphatic function in the K14sVEGFR-3-Fc mice was reflected by a significantly increased half-life (wildtype $=4.0 \pm 0.3 \mathrm{~h} ; \mathrm{K} 14-\mathrm{sVEGFR}-3-\mathrm{Fc}=$ $11.9 \pm 3.1 \mathrm{~h} ; P=0.002$; Supplementary Fig. 2c online) and significantly reduced clearance rate (wildtype $=$ $0.17 \pm 0.01 \mathrm{~h}^{-1} ; \quad$ K14-sVEGFR-3-Fc $=0.06 \pm 0.02 \mathrm{~h}^{-1}$; $P<0.001$; Supplementary Fig. 2d online). After injection of $5 \mu \mathrm{L}$ of $3 \mu \mathrm{M}$ P20D800 into the paw skin of K14-sVEGFR3 -Fc $(n=4)$ and wildtype littermates $(n=3)$, we again found a significant increase in tracer half-lives in the K14sVEGFR-3-Fc mice (wildtype $=2.4 \pm 0.6 \mathrm{~h}$; $\quad \mathrm{K} 14-$ sVEGFR-3-Fc $=13.5 \pm 3.4 \mathrm{~h} ; P=0.003$ ) with a corresponding decrease in the clearance rate (wildtype $=$ $0.31 \pm 0.09 \mathrm{~h}^{-1} ; \quad$ K14-sVEGFR-3-Fc $=0.06 \pm 0.02 \mathrm{~h}^{-1}$; $P=0.043$; Supplementary Fig. 3 online).

\section{Reduction of lymphatic clearance during aging}

We next applied the lymphatic clearance assay to investigate lymphatic function during aging. Three groups of female C57BL/6C mice were analyzed: young (2-months 
A

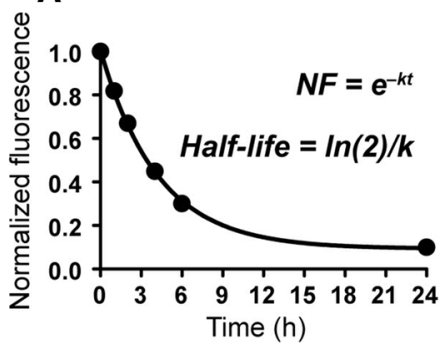

D

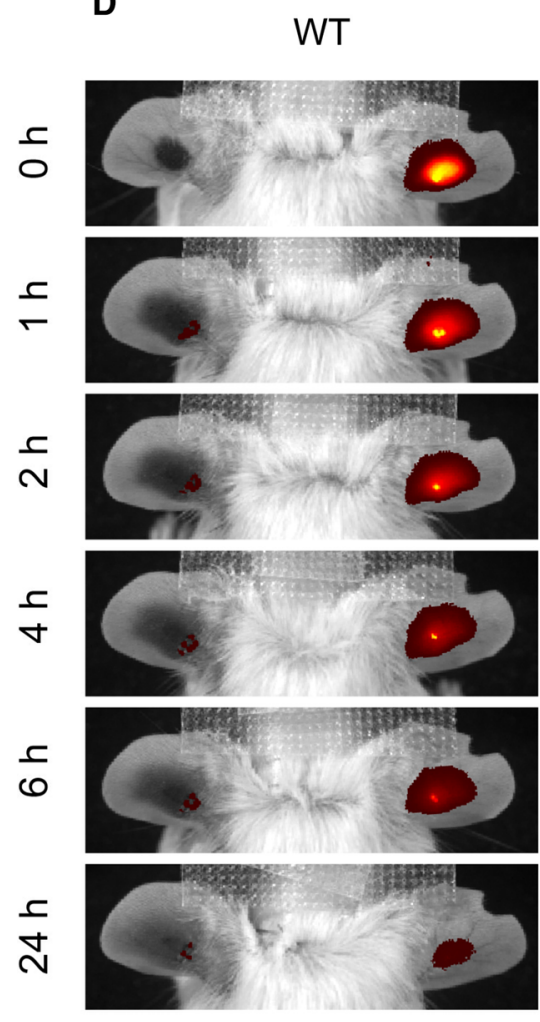

B

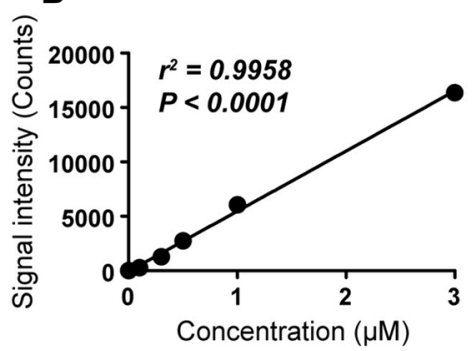

C

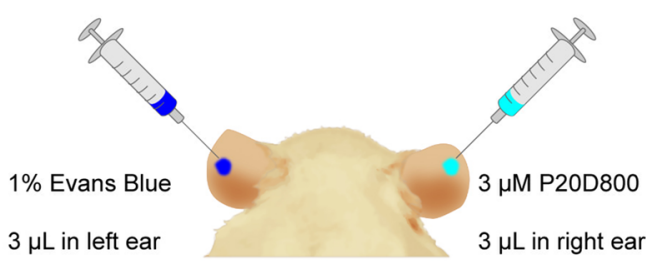

$\mathbf{E}$

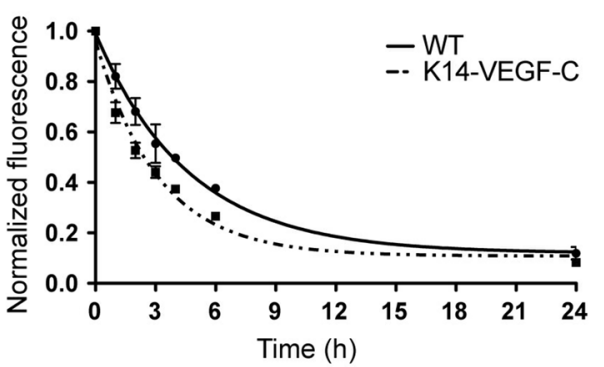

$\mathbf{F}$

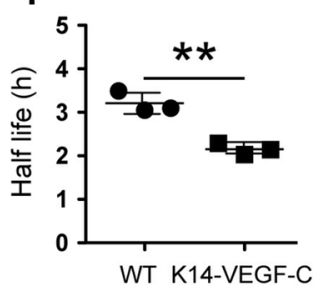

G

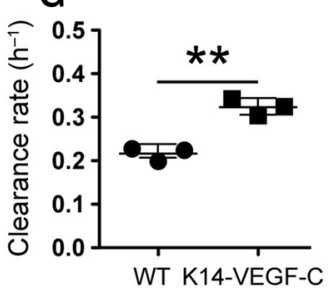

$\mathbf{H}$

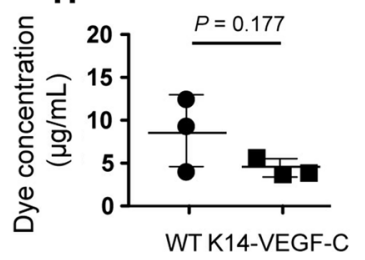

Fig. 1 Establishment of a clearance assay for lymphatic function in mouse skin. a The loss of fluorescence signal after injection of lymphatic specific fluorescent tracer should exhibit a one-phase exponential decay curve over time. Equations for the lymphatic clearance rate $\mathrm{k}$ and the half-life of clearance can be solved by plotting the normalized fluorescence (NF) intensity at several time points after injection (eg. 0, 1, 2, 4, 6, and 24 h). b Demonstration of the linear relationship between signal intensity and concentration of P20D800 as measured using an IVIS Spectrum. c Experimental setup to compare $1 \%$ Evans Blue versus P20D800 tracers for measurements of lymphatic clearance. d Representative images from sequential imaging of wildtype and K14-VEGF-C mice after injection of Evans Blue in the left ear and P20D800 into the right ear. e Normalized fluorescence intensity over time for wildtype and K14VEGF-C mice ( $\mathrm{n}=3$ each). Quantification of tracer half-lives (f) and clearance rates (g) in wildtype and K14-VEGF-C mice. Quantification of Evans blue dye concentration (h) at $24 \mathrm{~h}$ after dye extraction from ear skin. $* * P<0.01$ (two-tailed Student's $t$ test). Data are mean $\pm \mathrm{SD}$. (Color figure online)

$P=0.017)$ and young mice $(2.1 \pm 0.4 \mathrm{~h} ; P<0.001)$. The clearance rate (Fig. 2b) was significantly reduced in aged mice $\left(0.22 \pm 0.05 \mathrm{~h}^{-1}\right)$ when compared to young mice $\left(0.34 \pm 0.06 \mathrm{~h}^{-1} ; P<0.001\right)$.

This experiment was repeated with female mice of the same age groups in the C57BL/6 J-Tyr ${ }^{c-J}$ albino strain with similar findings (half-lives: young $=1.6 \pm 0.3 \mathrm{~h}$, middle-aged $=$ $2.2 \pm 0.6 \mathrm{~h}$, aged $=2.7 \pm 0.4 \mathrm{~h} ; P<0.01$ between young 

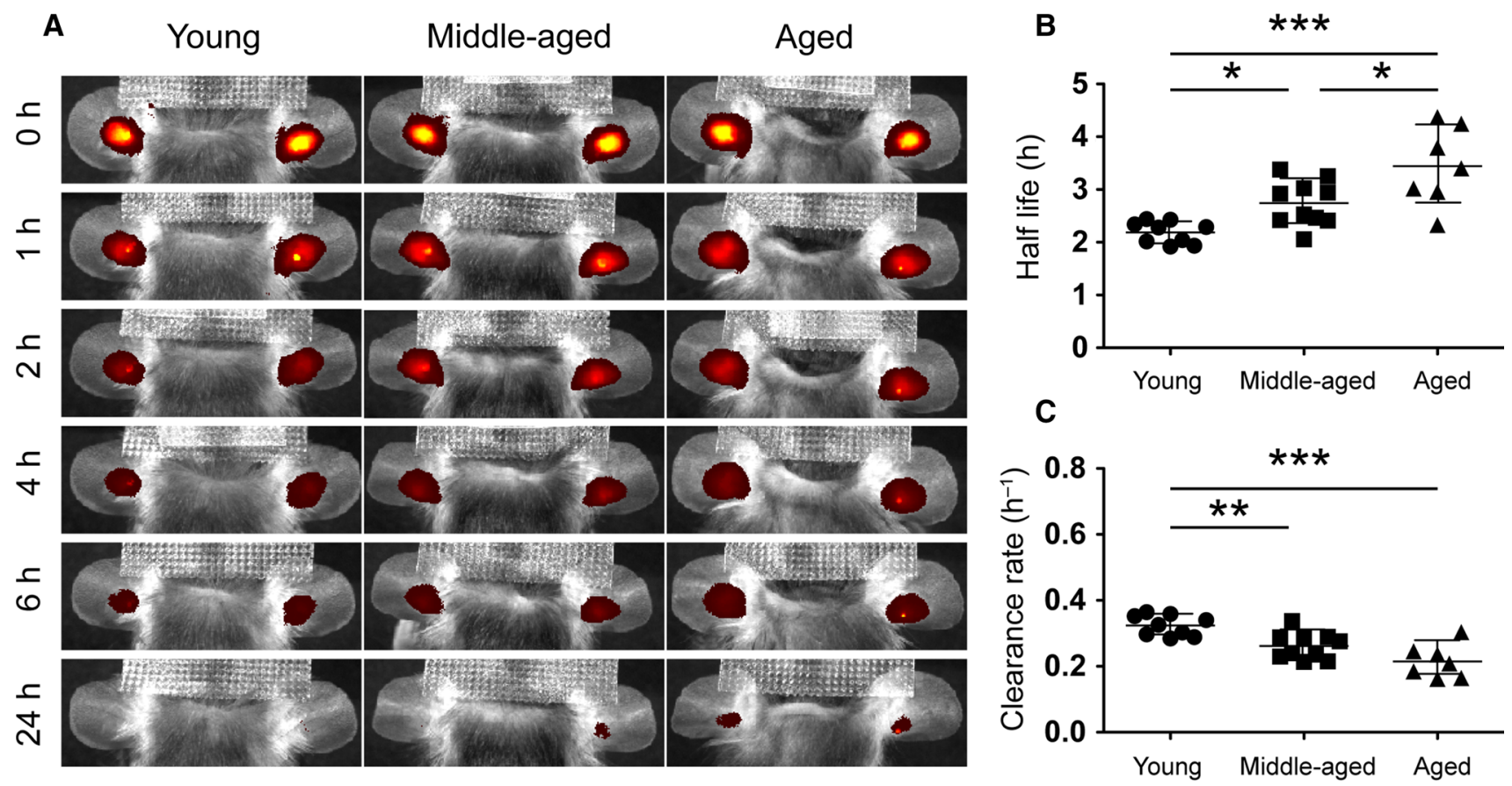

Fig. 2 Reduced lymphatic clearance from skin in aged mice. a Lymphatic specific fluorescent tracer $(3 \mu \mathrm{L}$ of $3 \mu \mathrm{M}$ P20D800) was injected into the skin of both ears of 2 months old (young, $\mathrm{n}=10$ ), 7 months old (middle-aged, $\mathrm{n}=10$ ), and 18 months old (aged, $\mathrm{n}=7$ ) mice. Sequential imaging was performed to track the

and aged groups). Together, these results reveal a consistent decline in lymphatic clearance during the aging process.

\section{Decreased lymphatic clearance during aging is not associated with a reduction of vascular leakage}

Since lymph formation is in large part driven by blood vascular capillary filtration we aimed to test if the decline in lymphatic function found in aged mice was associated with a reduction in vascular leakage. We measured blood vessel perfusion and leakage in the ears of young $(\mathrm{n}=6)$ and aged $(\mathrm{n}=5)$ C57BL/6 J-Tyr ${ }^{c-J}$ albino mice using a dynamic NIR imaging approach [18]. P20D800 was injected i.v. into the tail vein and the dynamics of fluorescence enhancement in the ear was compared to the fluorescence signal in the saphenous vein of the mice (Fig. 3a). Using this approach we found that there was no significant difference in dye perfusion into the ear blood vessels between young and aged mice (blood volume fraction: young $=0.079 \pm 0.012$, aged $=0.093 \pm 0.020$; $P=0.17$; Fig. 3b). However, aged mice had a significantly increased tissue leakage rate in the ears when compared to young mice (tissue leakage rate: young $=0.0014$ $\pm 0.0003 \mathrm{~min}^{-1}$, aged $=0.0022 \pm 0.0007 \mathrm{~min}^{-1} ; \quad P=$ 0.021; Fig. 3c). After normalization of the tissue leakage rate to the blood volume fraction the resultant vascular disappearance of the fluorescent signal. Quantification of half-lives (b) and clearance rates (c) in young, middle-aged and aged mice. $* P<0.05, \quad * * P<0.01, \quad * * * P<0.001$ (one-way ANOVA with Tukey's multiple comparison test). Data are mean \pm SD. (Color figure online)

leakage rate was also significantly increased in aged mice (vascular leakage rate: young $=0.0176 \pm 0.0028 \mathrm{~min}^{-1}$, aged $=0.0244 \pm 0.0066 \mathrm{~min}^{-1} ; \quad P=0.044 ; \quad$ Fig. $3 \mathrm{~d}$ ). These findings indicate that the reduced lymphatic clearance rate in aged mice after intradermal injection of tracers cannot be explained by a reduction of the steady-state vascular leakage rate with age.

\section{Decreased lymphatic vascular density and network complexity during aging}

We hypothesized that the decreased lymphatic clearance in aged mice might be related to the density of lymphatic vessels in the skin. Therefore, we performed whole mount stainings with the lymphatic vascular marker LYVE-1 on ears of young, middle-aged, and aged C57BL/6C mice (Fig. 4a). Quantification revealed that the area covered by LYVE-1 + lymphatic vessels $\quad$ (young $=0.188 \pm 0.014 \mathrm{~mm}^{2}, \quad$ middle-aged $=$ $0.166 \pm 0.020 \mathrm{~mm}^{2}, \quad$ aged $=0.149 \pm 0.021 \mathrm{~mm}^{2} ; \quad P=$ 0.002 between young and aged; Fig. $4 \mathrm{~b}$ ) as well as the total lymphatic vessel length per region of interest (Fig. 4c) were decreased during aging (young $=40.0 \pm 1.1 \mathrm{~mm}$, middleaged $=37.9 \pm 1.8 \mathrm{~mm}, \quad$ aged $=34.8 \pm 1.5 \mathrm{~mm} ; \quad P=$ 0.037 between young and middle-aged; $P<0.001$ between young and aged; $P=0.002$ between middle-aged and aged). The average vessel diameter was not significantly changed 

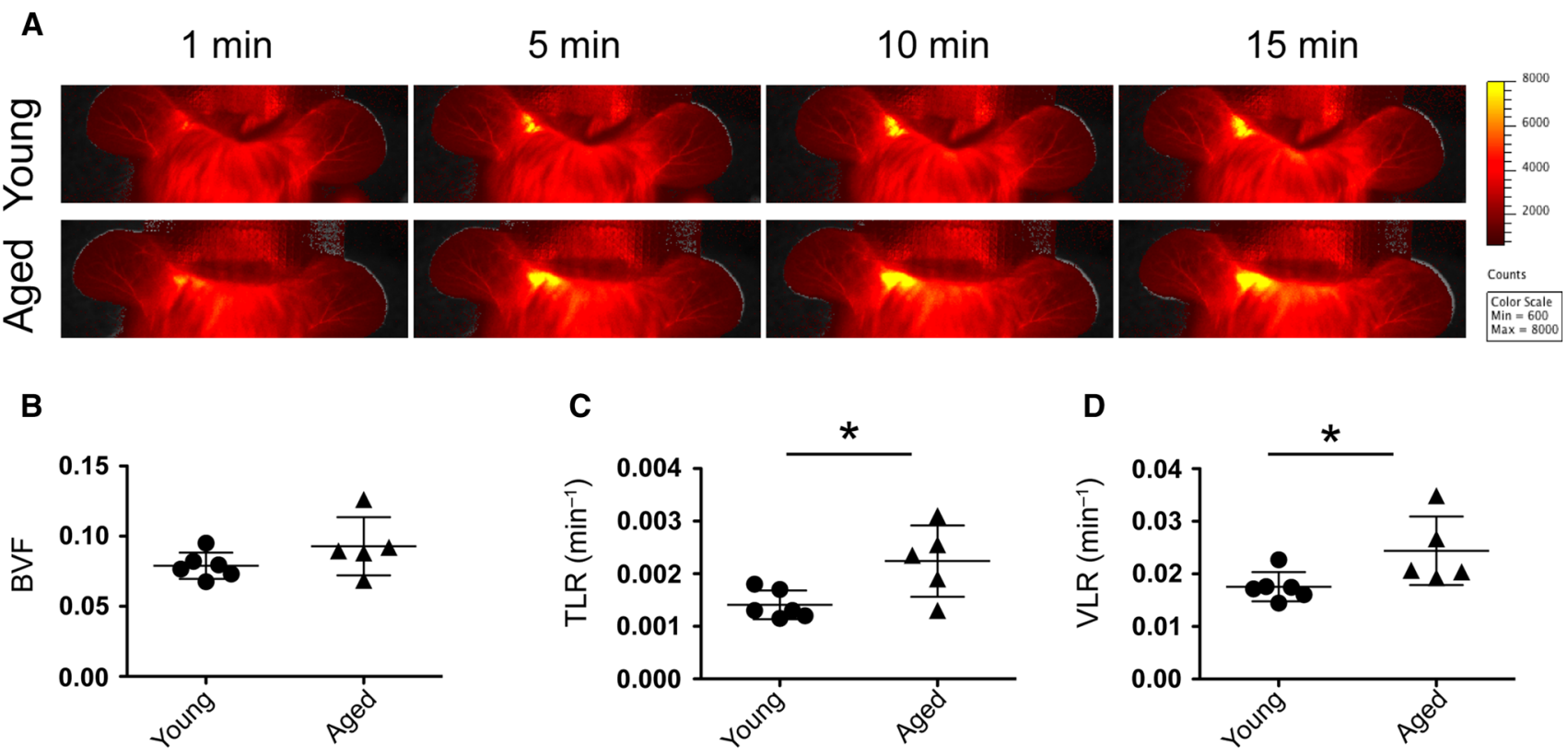

Fig. 3 Increased vascular leakage in ear skin of aged mice. a Representative images of the ears of 2 months old (young, $\mathrm{n}=6$ ) and 18 months old (aged, $\mathrm{n}=5$ ) C57BL/6 J-Tyr $r^{c-J}$ albino mice at 1 , 5,10 and $15 \mathrm{~min}$ after tail vein injection of P20D800. Sequential imaging was performed to calculate vascular leakage of the

during aging $\quad$ young $=47.1 \pm 3.7 \mu \mathrm{m}, \quad$ middle-aged $=$ $43.8 \pm 5.9 \mu \mathrm{m}$, aged $=42.7 \pm 5.4 \mu \mathrm{m} ; P=0.428$ between young and middle-aged; $P=0.267$ between young and aged; $P=0.903$ between middle-aged and aged; Fig. 4d), whereas the number of branch points per vessel length was decreased with aging (young $=2.4 \pm 0.4 \mathrm{~mm}^{-1}$, middle-aged $=2.0$ $\pm 0.3 \mathrm{~mm}^{-1}$, aged $=1.9 \pm 0.2 \mathrm{~mm}^{-1} ; P=0.019$ between young and middle-aged; $P=0.007$ between young and aged; $P=0.801$ between middle-aged and aged; Fig. 4e), indicating a reduction in the overall lymphatic vascular network complexity.

\section{Discussion}

In this study, we have established a method for the quantitative in vivo measurement of lymphatic clearance by tracking the disappearance of an injected, lymphaticspecific NIR fluorescent tracer from the skin of mice over time. To develop this functional assay, we adapted a method that has been established in the clinic using radiolabeled tracers [13]. In this procedure, a bolus of imaging tracer is injected into the skin and a rate removal constant $\mathrm{k}$ is calculated using serial imaging. For mice, a NIR lymphatic-specific tracer that we developed previously for visualization of collecting lymphatic vessel function was used [15]. We directly compared this technique to the established Evans Blue assay in which the amount of residual dye after a period of time can be fluorescent tracer. Quantification of blood volume fraction (b), tissue leakage rate (c) and vascular leakage rate (d) in young and aged mice. $* P<0.05, * * P<0.01, * * * P<0.001$ (two-tailed Student's $t$ test). Data are mean $\pm \mathrm{SD}$. (Color figure online)

calculated by extracting the dye from the tissue of sacrificed mice [20]. With the NIR method, we were able to detect a significantly increased lymphatic clearance function in K14-VEGF-C mice [16] versus wildtype controls with only three mice per group. By comparison, the difference between groups with the Evans Blue technique did not reach significance with this limited number of mice, due to an increased variability of the data points. Interestingly, K14-VEGF-C mice demonstrated an immediate spreading throughout the ear tissue after intradermal injection of both P20D800 and Evans blue. We confirmed using high-resolution lymphangiography that the spreading of tracer indeed occurs within the lymphatic vasculature of these mice with no apparent leakage into the interstitium. The expansion of a functional dermal lymphatic vessel network in these mice improved the clearance of intradermally injected tracers, most likely by increasing the available volume for absorption of macromolecules. The improved lymphatic function may be beneficial in K14-VEGF-C mice, as we have previously shown that these mice have reduced ear swelling in both acute and chronic inflammatory models compared to wildtype controls [20,26]. We also validated the method by demonstrating a significant reduction of lymphatic clearance from both ears and paws in K14sVEGFR-3-Fc mice [17]. These findings are in agreement with a recently reported study testing lymphatic function in the paws of these mice [25]. 
A
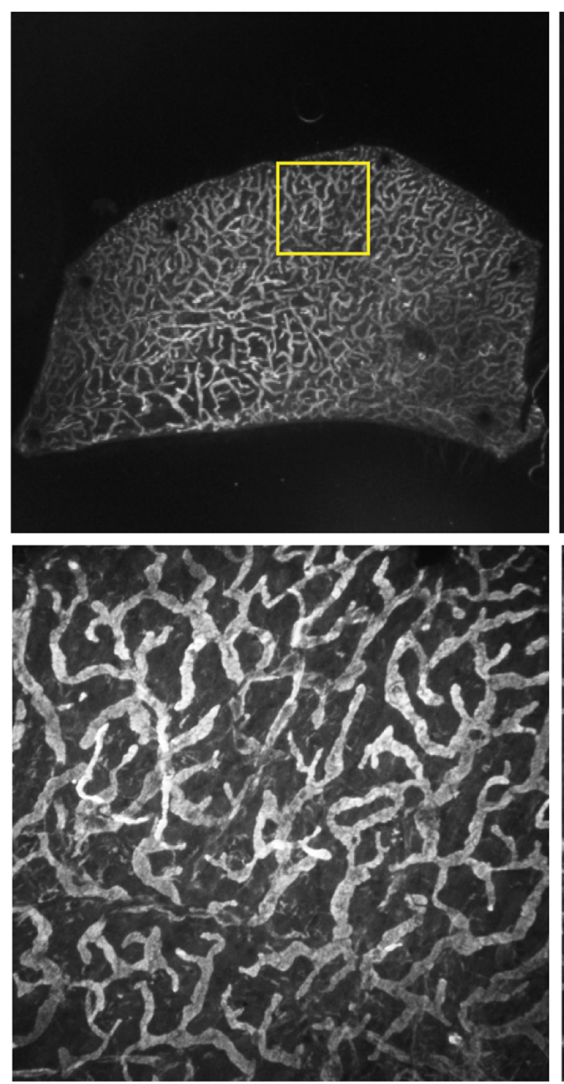

Middle-aged
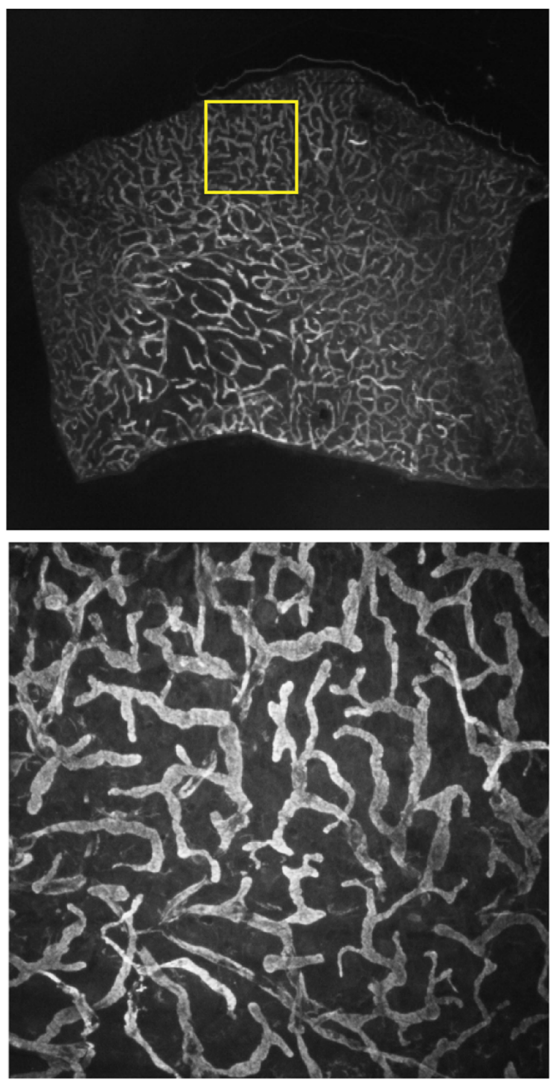

Aged
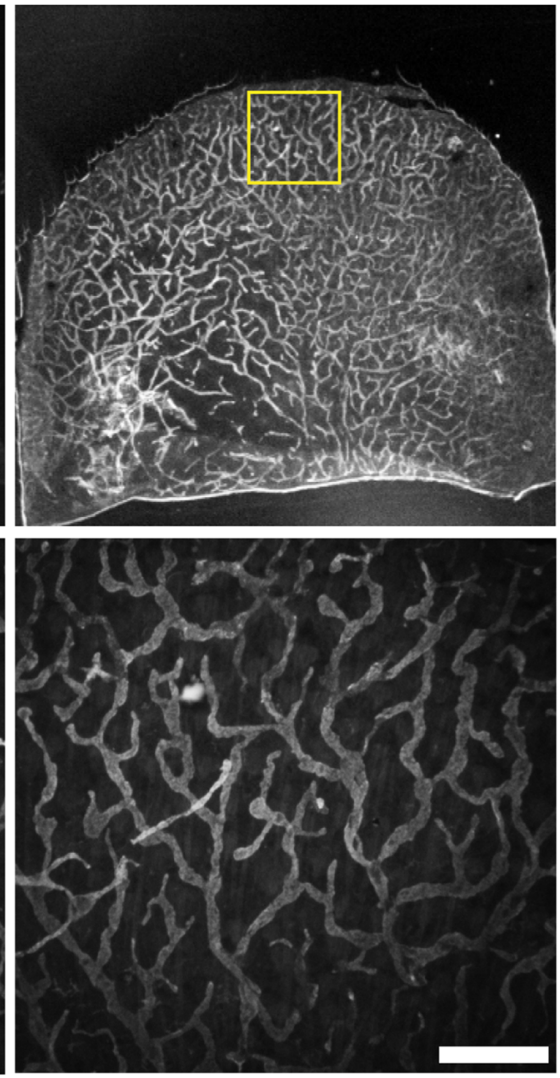

$500 \mu \mathrm{m}$

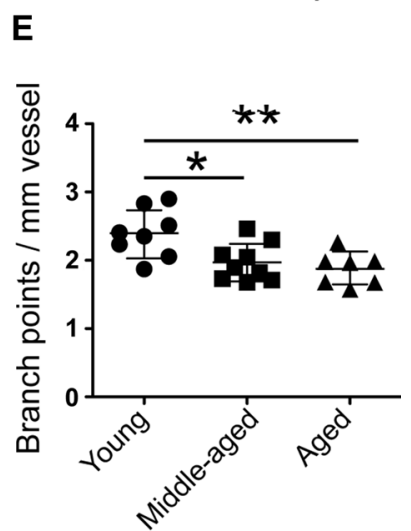

Fig. 4 Decreased lymphatic density and network complexity in skin of aged mice. a Whole mount preparations of ears were stained with anti-LYVE-1 antibody for visualization of the lymphatic network of the ear skin in young $(n=7)$, middle aged $(n=8)$ and aged $(n=7)$ mice. Images of the lymphatic network near the ear rim (yellow boxes in upper panels) were acquired using a fluorescence stereomicroscope

Among the many advantages of a fluorescence-based method are the ability to normalize the signal to a value directly after injection, the possibility to monitor the decay of signal over several time points allowing a curve fit to an exponential decay model, elimination of the need to sacrifice the animals for dye extraction allowing longitudinal
D

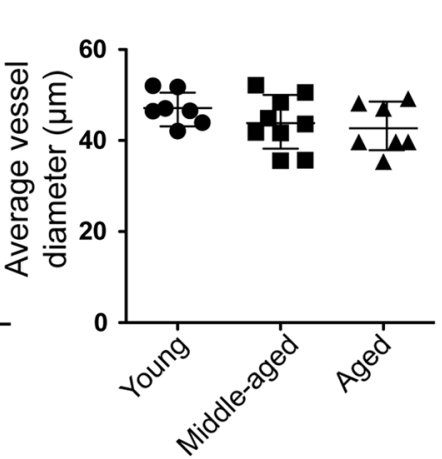

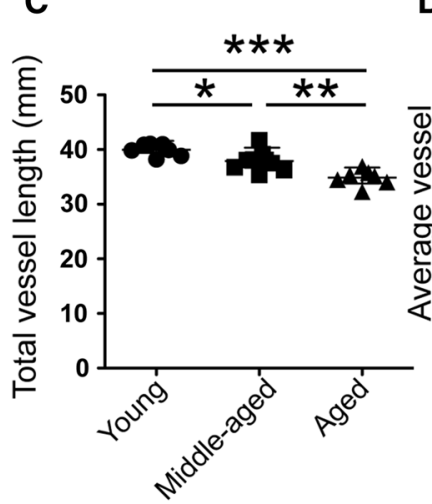

(lower panels, scale bar indicates $500 \mu \mathrm{m}$ ). Quantification of LYVE$1^{+}$area (b), total vessel length (c), average vessel diameter (d) and number of branch points per length of lymphatic vessel (e) in young, middle-aged and aged mice. $* P<0.05, * * P<0.01$, $* * * P<0.001$ (one-way ANOVA with Tukey's multiple comparison test). Data are mean $\pm \mathrm{SD}$. (Color figure online)

monitoring over time, and the capability to preserve the tissue for further analyses such as immunohistochemistry. Recently, a method was described using fluorescently labeled human serum albumin to track lymphatic clearance from the paws of mice [14]. This method has the potential advantage of monitoring the clearance of a serum protein 
commonly found in interstitial tissue; however, as pointed out by the authors, the use of human albumin in rodents may lead to an immune response that may hinder longitudinal studies [14].

We applied the NIR clearance assay to evaluate the lymphatic function in C57BL/6C mice of different age groups. The lymphatic clearance from the skin was decreased with aging, with significant increases of clearance half-lives in mice aged from 2 months (young) to 7 months (middle-aged) and from 7 months to 18 months (aged). As the lymphatic clearance assay measures the removal rate of an interstitially injected tracer, it could be affected by several factors. First, the vascular leakage from plasma could play a role, as it is the main driving force for the formation of lymph from interstitial fluid. The composition of the interstitium could affect the diffusion of fluid (and the convective transport of the tracer) through the tissue to reach the lymphatic vessels, and the density of the initial lymphatic vessels in the tissue could affect the uptake of lymph from the interstitium by changing the surface area available for absorption. Finally, changes may be seen in the collecting lymphatic vessel morphology and function that transport lymph back towards the blood circulatory system.

We first measured blood vascular leakage using NIR imaging in the ear skin of young versus aged mice. The results indicated that neither blood vascular density nor leakage was reduced with aging. On the contrary, we found that the vascular leakage was slightly increased in the aged skin similar to previous reports on murine heart and brain tissue [27, 28]. Therefore, we believe that a diminished capillary filtration cannot explain the reduced lymphatic clearance with aging.

We next focused our efforts to evaluate the effects of aging on the network of initial lymphatic vessels in the skin. Using whole mount stainings of ears for the lymphatic marker LYVE-1 to assess the density and morphology of initial lymphatic vessels, we found a significant reduction in lymphatic density in the skin of aged mice compared to young controls. These changes are similar to those reported in blood vessels and lymphatic vessels in the photoaged skin of humans $[29,30]$. While there was no difference in the diameter of the initial lymphatic vessels, a reduction of branching points was found in aged mice, indicating a reduced complexity of the network. These results indicate that involution may be occurring in the lymphatic vessel network of the skin during the aging process.

In conclusion, our findings reveal a decrease in lymphatic function in mouse skin during the aging process with a concomitant reduction of the density and complexity of the initial lymphatic network. Since many lymphatic-related diseases are more prevalent in aged individuals, this decline of function may have clinical implications for a range of conditions including autoimmune diseases, atherosclerosis, cancer, and lymphedema.

Acknowledgments The authors thank Jeannette Scholl and Carlos Ochoa for excellent technical assistance. This work was supported by Swiss National Science Foundation Grants 3100A0-108207 and 31003A-130627, Advanced European Research Council Grant LYVICAM, Oncosuisse, Krebsliga Zurich, and Leducq Transatlantic Network of Excellence on Lymph Vessels in Obesity and Cardiovascular Disease (11CVD03).

\section{Compliance with ethical standards}

Conflict of interest The authors declare that they have no conflict of interest.

Ethical approval All applicable international, national, and/or institutional guidelines for the care and use of animals were followed.

\section{References}

1. Alitalo K (2011) The lymphatic vasculature in disease. Nat Med 17(11):1371-1380. doi:10.1038/nm.2545

2. Proulx ST, Luciani P, Dieterich L, Karaman S, Leroux JC, Detmar M (2013) Expansion of the lymphatic vasculature in cancer and inflammation: new opportunities for in vivo imaging and drug delivery. J Control Release 172(2):500-507. doi:10.1016/j. jconrel.2013.04.027

3. Martel C, Li W, Fulp B, Platt AM, Gautier EL, Westerterp M, Bittman R, Tall AR, Chen SH, Thomas MJ, Kreisel D, Swartz MA, Sorci-Thomas MG, Randolph GJ (2013) Lymphatic vasculature mediates macrophage reverse cholesterol transport in mice. J Clin Invest 123(4):1571-1579. doi:10.1172/JCI63685

4. Lim HY, Thiam CH, Yeo KP, Bisoendial R, Hii CS, McGrath KCY, Tan KW, Heather A, Alexander JSJ, Angeli V (2013) Lymphatic vessels are essential for the removal of cholesterol from peripheral tissues by SR-BI-mediated transport of HDL. Cell Metab 17(5):671-684. doi:10.1016/j.cmet.2013.04.002

5. Wiig H, Schroder A, Neuhofer W, Jantsch J, Kopp C, Karlsen TV, Boschmann M, Goss J, Bry M, Rakova N, Dahlmann A, Brenner S, Tenstad O, Nurmi H, Mervaala E, Wagner H, Beck FX, Muller DN, Kerjaschki D, Luft FC, Harrison DG, Alitalo K, Titze J (2013) Immune cells control skin lymphatic electrolyte homeostasis and blood pressure. J Clin Invest 123(7):2803-2815. doi:10.1172/JCI60113

6. Ryan T (2004) The ageing of the blood supply and the lymphatic drainage of the skin. Micron 35(3):161-171. doi:10.1016/j. micron.2003.11.010

7. Mortimer PS (1995) Evaluation of lymphatic function: abnormal lymph drainage in venous disease. Int Angiol 14(3 Suppl 1):32-35

8. Conway WC, Faries MB, Nicholl MB, Terando AM, Glass EC, Sim M, Morton DL (2009) Age-related lymphatic dysfunction in melanoma patients. Ann Surg Oncol 16(6):1548-1552. doi:10. 1245/s10434-009-0420-x

9. Gasheva OY, Knippa K, Nepiushchikh ZV, Muthuchamy M, Gashev AA (2007) Age-related alterations of active pumping mechanisms in rat thoracic duct. Microcirculation 14(8):827-839. doi:10.1080/10739680701444065

10. Nagai T, Bridenbaugh EA, Gashev AA (2011) Aging-associated alterations in contractility of rat mesenteric lymphatic vessels. Microcirculation 18(6):463-473. doi:10.1111/j.1549-8719.2011. 00107.x 
11. Akl TJ, Nagai T, Cote GL, Gashev AA (2011) Mesenteric lymph flow in adult and aged rats. Am J Physiol Heart Circ Physiol 301(5):H1828-H1840. doi:10.1152/ajpheart.00538.2011

12. Unno N, Tanaka H, Suzuki M, Yamamoto N, Mano Y, Sano M, Saito T, Konno H (2011) Influence of age and gender on human lymphatic pumping pressure in the leg. Lymphology 44(3): $113-120$

13. Modi S, Stanton A, Mortimer P, Levick J (2007) Clinical assessment of human lymph flow using removal rate constants of interstitial macromolecules: a critical review of lymphoscintigraphy. Lymphat Res Biol 5(3):183-202

14. Karlsen TV, McCormack E, Mujic M, Tenstad O, Wiig H (2012) Minimally invasive quantification of lymph flow in mice and rats by imaging depot clearance of near-infrared albumin. Am J Physiol Heart Circ Physiol 302(2):H391-H401. doi:10.1152/ ajpheart.00842.2011

15. Proulx ST, Luciani P, Christiansen A, Karaman S, Blum KS, Rinderknecht M, Leroux JC, Detmar M (2013) Use of a PEGconjugated bright near-infrared dye for functional imaging of rerouting of tumor lymphatic drainage after sentinel lymph node metastasis. Biomaterials 34(21):5128-5137. doi:10.1016/j.bioma terials.2013.03.034

16. Jeltsch M, Kaipainen A, Joukov V, Meng X, Lakso M, Rauvala H, Swartz M, Fukumura D, Jain RK, Alitalo K (1997) Hyperplasia of lymphatic vessels in VEGF-C transgenic mice. Science 276(5317):1423-1425

17. Mäkinen T, Jussila L, Veikkola T, Karpanen T, Kettunen M, Pulkkanen K, Kauppinen R, Jackson D, Kubo H, Nishikawa S, Alitalo K (2001) Inhibition of lymphangiogenesis with resulting lymphedema in transgenic mice expressing soluble VEGF receptor-3. Nat Med 7:199-205

18. Proulx ST, Luciani P, Alitalo A, Mumprecht V, Christiansen AJ, Huggenberger R, Leroux JC, Detmar M (2013) Non-invasive dynamic near-infrared imaging and quantification of vascular leakage in vivo. Angiogenesis 34(21):5128-5137. doi:10.1007/ s10456-013-9332-2

19. Ott P, Keiding S, Bass L (1993) Plasma elimination of indocyanine green in the intact pig after bolus injection and during constant infusion: comparison of spectrophotometry and highpressure liquid chromatography for concentration analysis. Hepatology 18(6):1504-1515

20. Huggenberger R, Siddiqui SS, Brander D, Ullmann S, Zimmermann K, Antsiferova M, Werner S, Alitalo K, Detmar M (2011) An important role of lymphatic vessel activation in limiting acute inflammation. Blood 117(17):4667-4678. doi:10.1182/blood2010-10-316356
21. Karaman S, Hollmen M, Robciuc MR, Alitalo A, Nurmi H, Morf B, Buschle D, Alkan HF, Ochsenbein AM, Alitalo K, Wolfrum C, Detmar M (2015) Blockade of VEGF-C and VEGF-D modulates adipose tissue inflammation and improves metabolic parameters under high-fat diet. Mol Metab 4(2):93-105. doi:10.1016/j.mol met.2014.11.006

22. Iolyeva M, Karaman S, Willrodt AH, Weingartner S, Vigl B, Halin C (2012) Novel role for ALCAM in lymphatic network formation and function. FASEB J 27(3):978-990. doi:10.1096/fj. $12-217844$

23. Lohela M, Heloterä H, Haiko P, Dumont DJ, Alitalo K (2008) Transgenic induction of vascular endothelial growth factor- $\mathrm{C}$ is strongly angiogenic in mouse embryos but leads to persistent lymphatic hyperplasia in adult tissues. Am J Pathol 173(6):1891-1901. doi:10.2353/ajpath.2008.080378

24. Blum KS, Karaman S, Proulx ST, Ochsenbein AM, Luciani P, Leroux JC, Wolfrum C, Detmar M (2014) Chronic high-fat diet impairs collecting lymphatic vessel function in mice. PLoS ONE 9(4):e94713. doi:10.1371/journal.pone.0094713

25. Markhus CE, Karlsen TV, Wagner M, Svendsen OS, Tenstad O, Alitalo K, Wiig H (2013) Increased interstitial protein because of impaired lymph drainage does not induce fibrosis and inflammation in lymphedema. Arterioscler Thromb Vasc Biol 33(2):266-274. doi:10.1161/ATVBAHA.112.300384

26. Huggenberger R, Ullmann S, Proulx ST, Pytowski B, Alitalo K, Detmar M (2010) Stimulation of lymphangiogenesis via VEGFR-3 inhibits chronic skin inflammation. J Exp Med 207(10):2255-2269. doi:10.1084/jem.20100559

27. Yabluchanskiy A, Ma Y, Chiao YA, Lopez EF, Voorhees AP, Toba H, Hall ME, Han HC, Lindsey ML, Jin YF (2014) Cardiac aging is initiated by matrix metalloproteinase-9-mediated endothelial dysfunction. Am J Physiol Heart Circ Physiol 306(10):H1398-H1407. doi:10.1152/ajpheart.00090.2014

28. Pelegri C, Canudas AM, del Valle J, Casadesus G, Smith MA, Camins A, Pallas M, Vilaplana J (2007) Increased permeability of blood-brain barrier on the hippocampus of a murine model of senescence. Mech Ageing Dev 128(9):522-528. doi:10.1016/j. mad.2007.07.002

29. Chung JH, Yano K, Lee MK, Youn CS, Seo JY, Kim KH, Cho KH, Eun HC, Detmar M (2002) Differential effects of photoaging vs intrinsic aging on the vascularization of human skin. Arch Dermatol 138(11):1437-1442

30. Kajiya K, Kunstfeld R, Detmar M, Chung JH (2007) Reduction of lymphatic vessels in photodamaged human skin. J Dermatol Sci 47(3):241-243. doi:10.1016/j.jdermsci.2007.05.003 